\title{
Introduction to the Web-method and its applications
}

\author{
Klaus Höllig, Christian Apprich and Anja Streit* \\ Universität Stuttgart, Fachbereich Mathematik, Pfaffenwaldring 57, 70569 Stuttgart, Germany
}

Received 27 March 2003; accepted 2 February 2004

Communicated by Z. Wu and B.Y.C. Hon

\begin{abstract}
The Web-method is a meshless finite element technique which uses weighted extended B-splines (Web-splines) on a tensor product grid as basis functions. It combines the computational advantages of B-splines and standard mesh-based elements. In particular, degree and smoothness can be chosen arbitrarily without substantially increasing the dimension. Hence, accurate approximations are obtained with relatively few parameters. Moreover, the regular grid is well suited for hierarchical refinement and multigrid techniques. This article should serve as an introduction to finite element approximation with B-splines. We first review the construction of Web-bases and discuss their basic properties. Then we illustrate the performance of Ritz-Galerkin schemes for a model problem and applications in linear elasticity. Finally, we discuss several implementation aspects.
\end{abstract}

Keywords: finite element, meshless method, Web-spline, B-spline, weight function, stability

AMS subject classification: 65N30, 41A15, 74S05

\section{Introduction}

The finite element method has become the method of choice for solving many types of partial differential equations in engineering and physical sciences. Important applications include structural mechanics, fluid flow, thermodynamics, and electromagnetic fields [25]. The basic idea is very elegant and dates back to the classical work of Rayleigh, Ritz, and Galerkin almost a century ago. Guided by physical principles, an elliptic boundary value problem is stated in variational form:

$$
Q(u)=\frac{1}{2} a(u, u)-\lambda(u) \rightarrow \min , \quad u \in H .
$$

Usually, the quadratic functional $Q$ represents the total energy in the underlying model. The symmetric bilinear form $a$ and the linear form $\lambda$ correspond to contributions from internal and applied forces, and $H$ is a Hilbert space which incorporates the boundary conditions. A classical example is the analysis of elastic deformations, which has been the starting point of finite element analysis [1,24].

\footnotetext{
* Present address: Fraunhofer ITWM, 67663 Kaiserslautern, Germany.
} 
The Ritz-Galerkin method restricts the minimization of (1) to a finite-dimensional subspace

$$
V_{h}=\operatorname{span}_{i} B_{i} \subset H .
$$

Under mild assumptions, the resulting approximation

$$
u_{h}=\sum_{i} u_{i} B_{i} \approx u
$$

defined by

$$
Q\left(u_{h}\right)=\min _{v_{h} \in V_{h}} Q\left(v_{h}\right),
$$

will converge to $u$ as the discretization parameter $h$ (typically a grid width) tends to 0 . A crucial requirement is that the bilinear form is elliptic. In the symmetric case this means that $a$ induces an equivalent norm on $H$ and implies

$$
\left\|u-u_{h}\right\|_{H} \leqslant \operatorname{const}(a) \inf _{v_{h} \in V_{h}}\left\|u-v_{h}\right\|_{H} .
$$

This fundamental inequality, due to Céa, follows directly from the characterization of $u_{h}$ as the best approximation to $u$ in the $a$-norm [23].

The numerical computation of $u_{h}$ is relatively straightforward; the minimization of positive definite quadratic functionals is well understood. Primarily for very large systems or time-dependent problems more sophisticated techniques, which take advantage of the special structure of finite element equations, have to be applied.

Standard finite element subspaces $V_{h}$ consist of continuous piecewise polynomials on a partition of the simulation region $D$ into polygonal cells (triangles or quadrilaterals in two, and tetrahedra or hexahedra in three dimensions). Conceptually, this approach is quite simple. However, constructing good meshes can be rather time-consuming. In particular, in three dimensions automatic grid generation is often a bottleneck in finite element simulations. Moreover, on unstructured meshes, the lack of smoothness leads to a dramatic increase in the dimension of $V_{h}$ for higher degree approximations. Finally, the accuracy near the boundary is poor unless isoparametric elements are used.

The inherent difficulties in using piecewise polynomials on irregular meshes motivated intensive research on meshless methods (cf., e.g., [2,3,21] for an overview). Most of the new concepts, such as least squares methods or Lagrange multiplier techniques, circumvent the direct discretization of boundary conditions. Hence, they do not require meshes which conform to the boundary. The price paid is often a more elaborate theory. For example, error estimates cannot be as easily derived and stability problems have to be dealt with.

In contrast, the Web-method, introduced in [12,13,15], is a meshless finite element approximation which stays within the standard Ritz-Galerkin framework, proposing a 
new type of finite element subspace $V_{h}$. The basis functions $B_{i}$ are weighted extended B-splines (Web-splines) on a tensor product grid and meet all basic finite element requirements. More precisely,

$$
B_{i}=w \sum_{k} e_{i, k} b_{k},
$$

where $w$ is a positive weight function and the sum is a linear combination of few neighboring $\mathrm{B}$-splines $b_{k}, k \approx i$. Choosing $w$ appropriately, homogeneous boundary conditions can be matched while keeping the simplicity of the tensor product spline space. The extension coefficients $e_{i, k}$ are needed to stabilize the basis. With the simple choice $B_{i}=w b_{i}$, the support of basis functions near the boundary can become very small, causing severe numerical problems.

Figure 1 shows a two-dimensional example for a uniform grid and Dirichlet boundary conditions. In this case $w$ vanishes on the entire boundary $\partial D$. As is typical, $B_{i} \approx b_{i}$ for B-splines in the interior of the domain; the effect of the weight function is hardly visible. Also, the linear combinations with coefficients $e_{i, k}$, which are formed near the boundary, do not have significantly larger support. Hence, the weight function and the extensions do not affect the qualitative structure of a standard spline space. Except for positivity (cf. the right Web-spline in the figure), all properties of B-splines are preserved.

The details of the construction of Web-splines are described in section 2. We then show in section 3 that the Web-basis possesses the familiar approximation properties. In section 4 we consider Laplace's equation in order to illustrate the Web-method in a simple setting and to compare the performance with standard techniques. Then we discuss in sections 5 and 6 problems in linear elasticity as typical engineering applications. Finally, section 7 is devoted to implementation aspects.

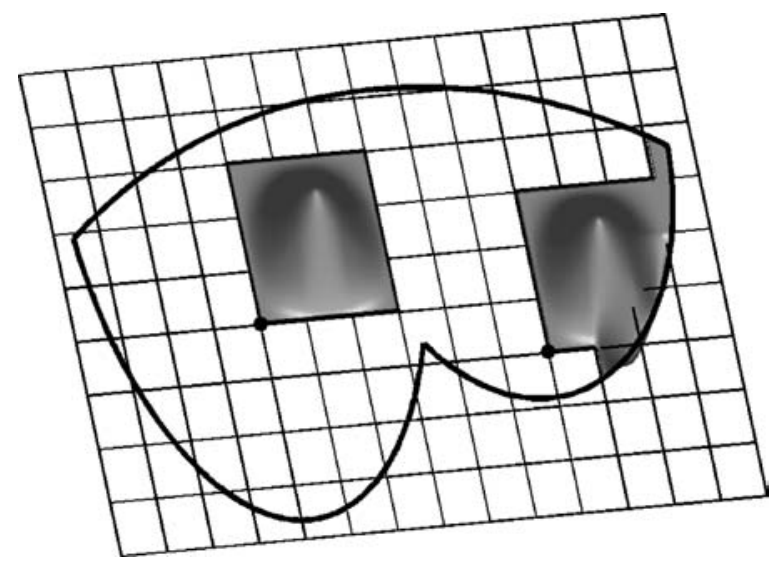

Figure 1. Biquadratic Web-splines $B_{i}$ for homogeneous Dirichlet boundary conditions, marked at the lower left corners $\left(i_{1}, i_{2}\right) h$ of the supports of the B-splines $b_{i}$. 
Notation. Throughout the paper we will use the following notational conventions.

For a bounded Lipschitz domain $D$ we denote by $H^{\ell}, \ell \in \mathbb{N}_{0}$, the Sobolev space of functions with square integrable derivatives up to order $\ell$, equipped with the norm

$$
\|u\|_{\ell}=\left(\sum_{|\alpha| \leq \ell} \int_{D}\left|\partial^{\alpha} u\right|^{2}\right)^{1 / 2}, \quad \partial^{\alpha}=\partial_{1}^{\alpha_{1}} \ldots \partial_{m}^{\alpha_{m}}
$$

(cf. [4]). Moreover, $H_{0}^{\ell}$ is the closure of smooth functions with compact support in $D$ with respect to the Sobolev norm.

In estimates, the dependence of constants on parameters $p_{v}$ is indicated in the form const $\left(p_{1}, p_{2}, \ldots\right)$. If the dependence is clear from the context, or not particularly relevant, we use the symbols $\preceq, \succeq$ and $\asymp$. For example,

$$
\operatorname{dist}(x, \partial D) \succeq h
$$

characterizes all points $x$ with distance $\geqslant$ const $h$ from the boundary of $D$.

Finally, $\|\cdot\|$ denotes the 2-norm of vectors and matrices.

\section{Web-basis}

Web-splines are modified B-splines which are adapted to standard finite element requirements. The formal definition (3) in the introduction involves a weight function $w$ and extension coefficients $e_{i, k}$. We discuss each of these components in turn after reviewing some basic definitions.

We denote by

$$
b_{k}, \quad k=\left(k_{1}, \ldots, k_{m}\right) \in \mathbb{Z}^{m},
$$

the $m$-variate tensor product B-spline of coordinate degree $n$ and support

$$
k h+[0, n+1]^{m} h,
$$

normalized, so that $\sum_{k} b_{k}=1$ (cf. figure 2). As is well known, the B-splines are linearly independent and the basis is uniformly stable with respect to the grid width $h[8,20]$.

For a bounded domain $D$,

$$
\mathbb{B}_{h}=\operatorname{span}_{k \in K} b_{k}
$$

denotes the linear span of all B-splines which have some support in $D$. This spline space provides in the usual way optimal order approximations for smooth functions. To incorporate homogeneous boundary conditions on a subset $\Gamma \subset \partial D$, we follow an old suggestion of Kantorovich and Krylow [16], which has been extensively studied by Rvachev and his co-authors (cf., e.g., the survey [19]). We multiply the B-splines $b_{k}$ by a positive weight function $w$, which vanishes on $\Gamma$ and denote the corresponding spline space by

$$
w \mathbb{B}_{h} .
$$




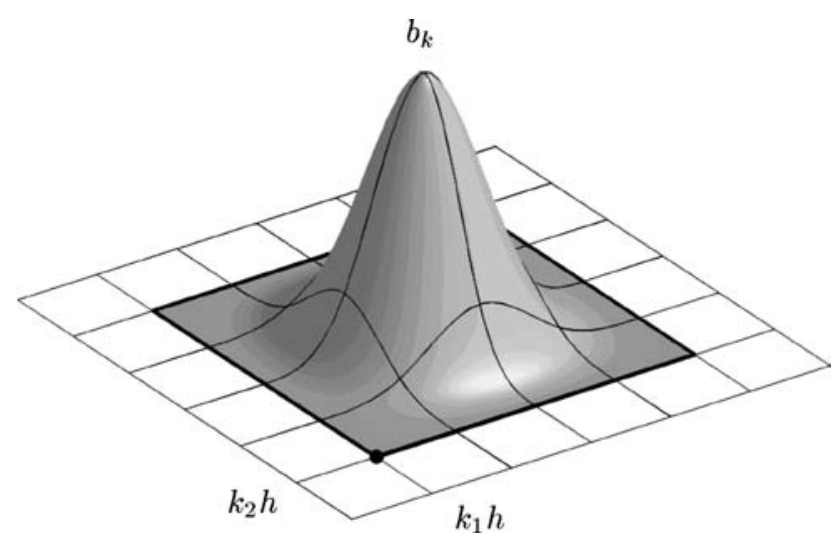

Figure 2. Tensor product grid and bivariate cubic B-spline $b_{k}(m=2, n=3)$, marked at the lower left corner $k h$ of its support.
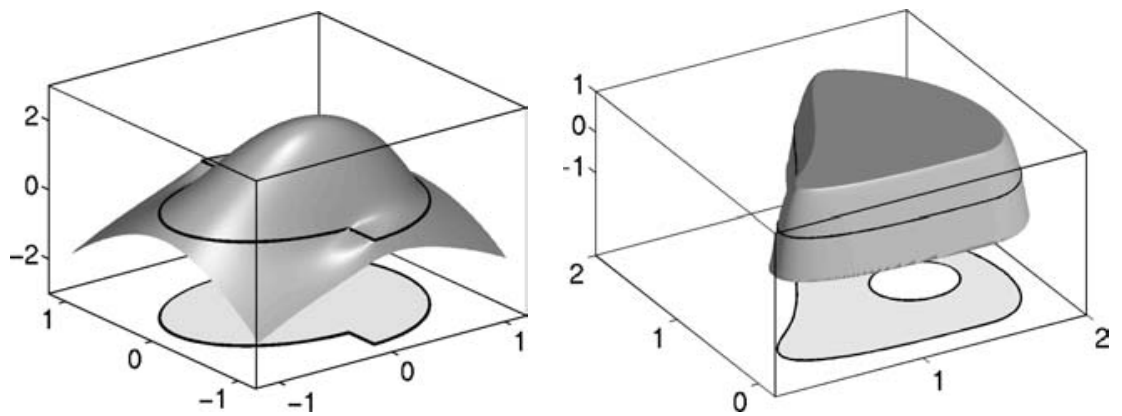

Figure 3. R-function (left) and blended distance function (right).

This is a very flexible approach. It does not only eliminate the need of boundary conforming elements but also allows us to utilize a priori knowledge about the qualitative behavior of the approximated functions. Clearly, if $w \approx u$, the numerical solution will be very accurate already for a moderate grid width.

There are several different techniques for constructing weight functions. Two examples are shown in figure 3 .

Rvachev's R-function method provides weight functions for domains which are defined via Boolean operations from simple primitives. The left side of the figure illustrates this for $\Gamma=\partial D$ and the domain

$$
D=\left(D_{0} \cap D_{1}\right) \cup D_{2}, \quad D_{i}=\left\{x: w_{i}>0\right\},
$$

with

$$
w_{0}=1-x_{1}^{2}-x_{2}^{2}, \quad w_{1}=x_{2}, \quad w_{2}=1-2 x_{1}^{2}-x_{2}^{2} .
$$


The set operations $\cap, U$ are translated into algebraic expressions via so-called R-functions $r_{\cap}, r_{\cup}$ :

$$
\begin{aligned}
w_{0,1} & =r_{\cap}\left(w_{0}, w_{1}\right)=w_{0}+w_{1}-\sqrt{w_{0}^{2}+w_{1}^{2}}, \\
w & =r_{\cup}\left(w_{0,1}, w_{2}\right)=w_{0,1}+w_{2}+\sqrt{w_{0,1}^{2}+w_{2}^{2}}
\end{aligned}
$$

(cf. [19] for details). This algorithmic approach combines particularly well with simulations for constructive solid geometry models, hence covers many typical engineering applications.

The right side of the figure shows a numerically constructed weight function $w$ for a domain with a free-form boundary. In this example, $\Gamma$ is a proper subset of $\partial D$ (the outer boundary) and $w$ is defined by blending the distance function with a plateau of height 1 in a $\delta$-neighborhood of $\Gamma$ via

$$
w(x)=1-\max \left(0,1-\frac{\operatorname{dist}(x, \Gamma)}{\delta}\right)^{\gamma} .
$$

The parameter $\delta$ must be chosen smaller than the minimal radius of curvature to avoid singularities of $\operatorname{dist}(\cdot, \Gamma)$, and $\gamma$ controls the smoothness of the blend.

Both types of weight functions can be evaluated efficiently. Moreover, several variants of the two basic methods are also available. For example, one can localize the construction of $w$ with the aid of a B-spline blending technique (cf. [10] for details).

We now turn to the discussion of the stabilization procedure. It is intuitively clear that the B-splines

$$
b_{j}, \quad j \in J \subset K,
$$

which do not have at least one grid cell of their support in $D$, can cause stability problems. This difficulty is overcome by suitably adjoining these so-called outer B-splines to the inner B-splines

$$
b_{i}, \quad i \in I=K \backslash J,
$$

which constitute the stable part of the basis. Of course, the approximation power of the spline space must be retained. Equivalently, the coefficients $e_{i, k}$ in definition (3) have to be chosen so that all weighted polynomials

$$
w p, \quad \text { degree } p \leqslant n,
$$

can be written as linear combinations of the Web-splines $B_{i}$. We state without proof the proper formula, which was derived in [12]. We select for each outer index $j$ an array

$$
I(j)=\ell(j)+\{0, \ldots, n\}^{m} \subset I
$$




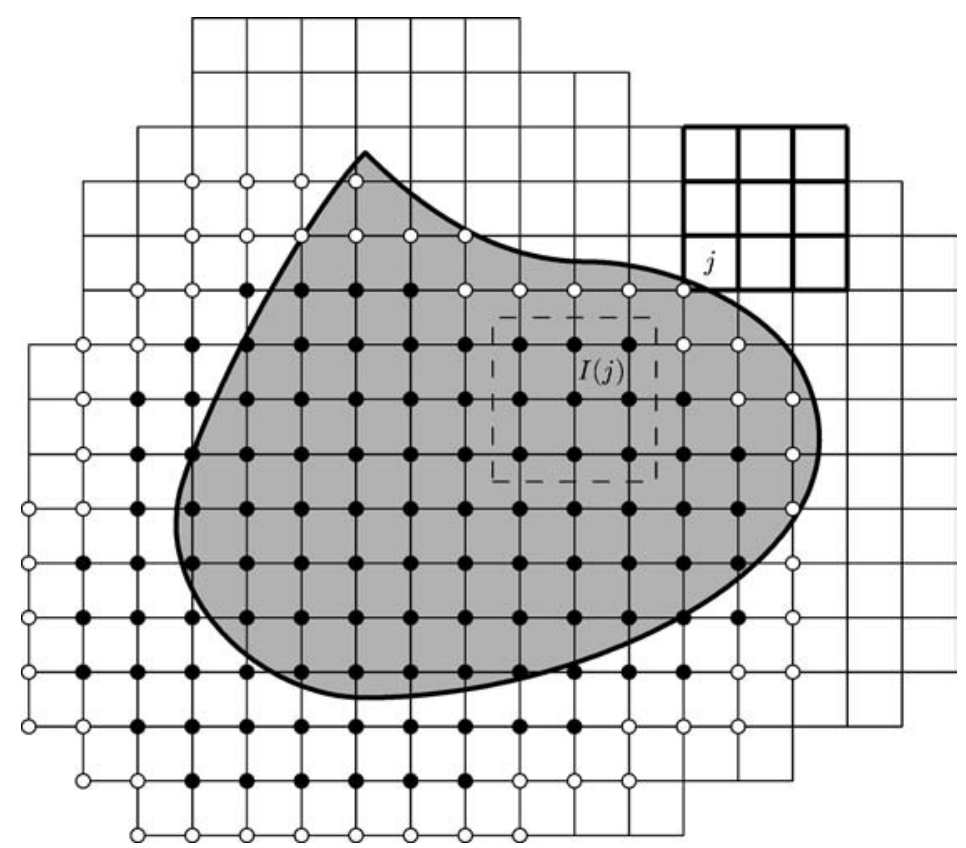

Figure 4. Inner and outer B-splines, marked with dots and circles, for a biquadratic spline space and an array $I(j)$ (enclosed by a dashed square) corresponding to an outer index $j$.

of inner indices closest to $j$ (cf. figure 4) and set

$$
e_{i, k}=\gamma_{i} \begin{cases}\prod_{\substack{\nu \\ \nu=1}}^{m} \prod_{\substack{\mu=0 \\ \ell_{\nu}+\mu \neq i_{\nu}}}^{n} \frac{k_{\nu}-\ell_{\nu}-\mu}{i_{\nu}-\ell_{\nu}-\mu} & \text { for } k=j \in J, i \in I(j) ; \\ 1 & \text { for } k=i \in I ; \\ 0 & \text { otherwise, }\end{cases}
$$

where $\gamma_{i}>0$ is a suitable normalization constant which ensures that

$$
\max _{x}\left|B_{i}(x)\right| \asymp 1 .
$$

For example, we may take $\gamma_{i}=1 / w\left(x_{i}\right)$ with $x_{i}$ the center of a grid cell $Q_{i} \subset \bar{D}$ in the support of $b_{i}$.

With the above definitions, we restate definition (3) more precisely as follows.

Definition 1 (Web-splines). The Web-splines for a bounded domain $D$, a positive weight function $w$, and extension coefficients $e_{i, k}$ are defined as

$$
B_{i}=w \sum_{k} e_{i, k} b_{k}, \quad i \in I,
$$

and form a basis for the Web-space $w^{e} \mathbb{B}_{h}$. 


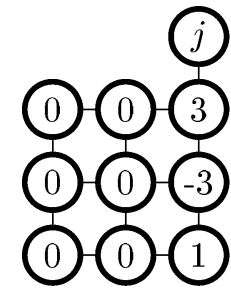

$I(j)$

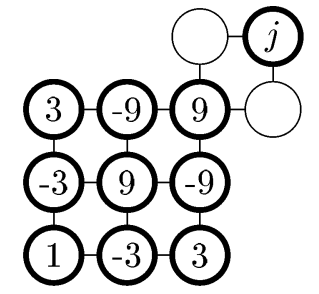

$I(j)$

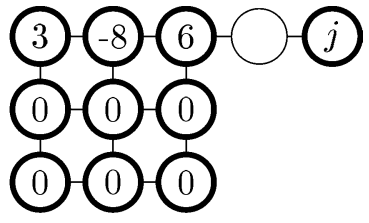

$I(j)$

Figure 5. Extension coefficients $e_{i, j} / \gamma_{i}, i \in I(j)$, for $m=n=2$ and several relative positions of $j$ and $I(j)$.

It is clear from the definition of the index arrays $I(j)$ that B-splines with support outside of a small boundary strip are not extended:

$$
B_{i}=\gamma_{i} w b_{i}, \quad \operatorname{dist}(i h, \Gamma) \geqslant \operatorname{const}(D, m, n) h .
$$

Hence, the percentage of nontrivial linear combinations is small. Moreover, from definition (4) we see that, up to normalization, the extension coefficients only depend on the relative position of the array $I(j)$ with respect to the outer index $j$. Typically, only a limited number of configurations occur, so that tabulated values can be used to generate the extension matrices $E$.

Figure 5 shows a few coefficient arrays for a biquadratic Web-space. The large number of zeros is typical. All coefficients $e_{i, j}, i \in I(j)$, are nonzero only if

$$
j_{v} \notin \ell_{v}+\{0, \ldots, n\} \quad \forall v=1, \ldots, m .
$$

Therefore, the linear combinations in the definition of $B_{i}$ involve usually only very few terms. This is also apparent from figure 1, which shows that the support of a Web-spline $B_{i}$ is generally not significantly larger than the support of the associated $\mathrm{B}$-spline $b_{i}$. Hence, the percentage of off-diagonal entries in $E$ is quite small.

\section{Approximation with Web-splines}

As is to be expected, Web-splines inherit all basic properties of B-splines. However, some mild assumptions on the weight function are necessary. With our primary applications in mind, we will consider weight functions of order $\gamma \in \mathbb{N}$, i.e.,

$$
w(x) \asymp \operatorname{dist}(x, \Gamma)^{\gamma}, \quad x \in D,
$$

for a sufficiently regular subset $\Gamma$ of $\partial D$ with positive $(m-1)$-dimensional measure. Moreover, we require that $w$ is $\ell$-regular for some $\ell>0$, i.e., the partial derivatives up to order $\ell$ are bounded and

$$
\left|\partial^{\alpha} w(x)\right| \leqslant \operatorname{const}(w) \operatorname{dist}(x, \Gamma)^{\gamma-|\alpha|}, \quad|\alpha| \leqslant \min (\gamma, \ell) .
$$


A standard case is $\Gamma=\partial D, \gamma=1$, and $\ell=1$ which corresponds to Dirichlet boundary conditions and minimal regularity. Also needed later on for the plate problem is $\gamma=2$. In this case, the weight function vanishes together with its normal derivative on $\Gamma$.

With the above assumptions, we can show that the Web-basis is well conditioned, uniformly with respect to the grid width $h$.

Theorem 1 (Stability). For an $\ell$-regular weight function of order $\gamma$

$$
h^{\nu}\left\|u_{h}\right\|_{v} \preceq\left\|u_{h}\right\|_{0} \asymp h^{m / 2}\|U\|, \quad v \leqslant \ell \leqslant n,
$$

for $u_{h}=\sum_{i \in I} u_{i} B_{i}$ and $U=\left\{u_{i}\right\}_{i \in I}$. In particular, $\left\|B_{i}\right\|_{\ell} \preceq h^{m / 2} h^{-\ell}$.

The proof is somewhat technical, but routine. The main tool are dual functions $\Lambda_{i}$ which vanish outside the support of $B_{i}$ and satisfy

$$
\int \Lambda_{i} B_{i^{\prime}}=\delta_{i, i^{\prime}}, \quad\left\|\Lambda_{i}\right\|_{0} \asymp h^{-m / 2} .
$$

For their construction we observe that none of the outer B-splines $b_{j}$ have support on the grid cell $Q_{i} \subset \bar{D} \cap \operatorname{supp} b_{i}$ with center $x_{i}$. Hence,

$$
B_{i}(x)=\gamma_{i} w(x) b_{i}(x), \quad x \in Q_{i},
$$

so that, with appropriate modifications, dual functions $\lambda_{i}$ for the standard B-splines can be used. We define

$$
\Lambda_{i}=\frac{1}{\gamma_{i} w} \lambda_{i}
$$

where the support of $\lambda_{i}$ has to be chosen in a subcell of $Q_{i}$ so that $w$ is nonzero. Details of the arguments can be found in [10].

With the aid of the dual functions we define a canonical projector

$$
P_{h} u=\sum_{i}\left(\int \Lambda_{i} u\right) B_{i}
$$

onto the Web-space $w^{e} \mathbb{B}_{h}$. While not of any practical relevance, it is a convenient operator for deriving error estimates. Since $P_{h}(w p)=w p$ for any polynomial $p$ of coordinate degree $\leqslant n$ and the coefficients $\left(\int \Lambda_{i} u\right)$ are computed from local information, it follows that Web-splines approximate with optimal order.

Theorem 2 (Approximation order). For an $\ell$-regular weight function $(\ell \leqslant n)$ of order $\gamma$,

$$
\left\|u-P_{h} u\right\|_{\ell} \leqslant \operatorname{const}(u, D, w, n) h^{n+1-\ell},
$$

if $v=u / w$ is smooth on $\bar{D}$.

Proof. This estimate is proved by analyzing the error separately on each grid cell $Q$. To this end we denote by $Q^{\prime}$ a smallest $m$-dimensional cube containing the support of 
all Web-splines which are nonzero on $Q$. Moreover, we write $u=w v$ and let $v^{\prime}$ be a smooth extension of $v$ to $\mathbb{R}^{m}$ as described in [22].

The key argument relates the error $u-P_{h} u$ on $D \cap Q$ to

$$
e=w\left(v^{\prime}-p\right)
$$

where $p$ is the best polynomial approximation of coordinate degree $\leqslant n$ to $v^{\prime}$ on $Q^{\prime}$ with respect to the norm $\|\cdot\|_{\ell}$. Since $P_{h}$ is exact for the weighted polynomial $w p$ and uses only information from within the domain $D$, where $v=v^{\prime}$,

$$
u-P_{h} u=w v^{\prime}-w p+P_{h}(w p)-P_{h}\left(w v^{\prime}\right)=e-P_{h} e
$$

on $D$. We estimate each term in turn. Since $w$ has bounded partial derivatives up to order $\ell$,

$$
\|e\|_{\ell, Q \cap D} \preceq\left\|v^{\prime}-p\right\|_{\ell, Q^{\prime}} .
$$

To bound the norm of the projection $P_{h} e=\sum_{i}\left(\int \Lambda_{i} e\right) B_{i}$, we note that on $Q \cap D$ only $\preceq 1$ summands are nonzero. Referring to the corresponding indices with $i \sim Q$, we have

$$
\operatorname{supp} \Lambda_{i} \subset Q^{\prime} \cap D, \quad i \sim Q,
$$

and it follows that

$$
\begin{aligned}
\left\|P_{h} e\right\|_{\ell, Q \cap D} & \preceq \max _{i \sim Q}\left\|\Lambda_{i}\right\|_{0}\|e\|_{0, Q^{\prime} \cap D}\left\|B_{i}\right\|_{\ell} \\
& \preceq h^{-m / 2}\left\|v^{\prime}-p\right\|_{0, Q^{\prime}} h^{m / 2} h^{-\ell},
\end{aligned}
$$

where we have used the boundedness of $w$ and theorem 1 for the second inequality. Hence, it remains to estimate the error $v^{\prime}-p$ of the polynomial approximation. Invoking the Bramble-Hilbert lemma, we obtain

$$
\left\|v^{\prime}-p\right\|_{v, Q^{\prime}} \preceq h^{n+1-v}\left\|v^{\prime}\right\|_{n+1, Q^{\prime}} .
$$

We apply this estimate with $v=\ell, 0$ to (6) and (7) and obtain

$$
\left\|u-P_{h} u\right\|_{\ell, Q \cap D}^{2} \preceq h^{2(n+1-\ell)}\left\|v^{\prime}\right\|_{n+1, Q^{\prime}}^{2}
$$

Summing this inequality over all grid cells $Q$ and noting that the corresponding $m$-cubes $Q^{\prime}$ overlap only $\preceq 1$ times completes the proof.

Theorem 2 assumes that $u / w$ is smooth. Estimates with minimal regularity are much harder to derive. Since $w v \in H^{n+1}$ does not imply that $v \in H^{n+1}$, even if $w$ is smooth, a subtle analysis of the quotient $u / w$ is necessary. For smooth weight functions of order 1 it was shown in $[10,12]$ that

$$
\left\|u-P_{h} u\right\|_{\ell} \leqslant \operatorname{const}(D, w, n) h^{k-\ell}\|u\|_{k}, \quad \ell<k \leqslant n+1, u \in H^{n+1} \cap H_{0}^{1},
$$

and it is likely that this result extends to weight functions of higher order. 
In view of Céa's inequality (2), theorem 2 implies a corresponding result for the error of Ritz-Galerkin approximations with $V_{h}=w^{e} \mathbb{B}_{h}$. Since for typical elliptic boundary value problems, the norm on the Hilbert space $H$ can be bounded in terms of a Sobolev norm $\|\cdot\|_{\ell}(\ell=1$ for second order problems $)$,

$$
\left\|u-u_{h}\right\|_{H} \preceq h^{n+1-\ell}
$$

for smooth solutions $u$.

We emphasize that regularity of solutions, as assumed in the above estimate, does not follow from the variational approach, but requires more subtle techniques. However, from a numerical point of view it is legitimate to focus entirely on the finite element approximation, based on the given properties of $u$.

It should also be noted that the optimal rate is not always attained. For solutions $u$ with singularities, caused, e.g., by corners and edges of the domain, the approximation order can deteriorate substantially. Here, adaptive refinement with hierarchical B-splines or special basis functions with singular weights is needed to avoid a significant loss of accuracy. Obviously, both techniques combine well with the Web-method.

\section{Model problem}

As a first illustration of the Web-method, we consider an elementary heat conduction problem. This application exhibits already some essential features of more general elliptic systems and serves as a test case for various error estimates.

As is depicted in figure 6, two pipes with radius $r$, distance $2 r$, and constant temperature $u_{0}$ are enclosed in a cylindrical insulation with cross section

$$
D_{\mathrm{c}}: \quad \frac{x_{1}^{2}}{16}+\frac{x_{2}^{2}}{9}<r^{2}
$$

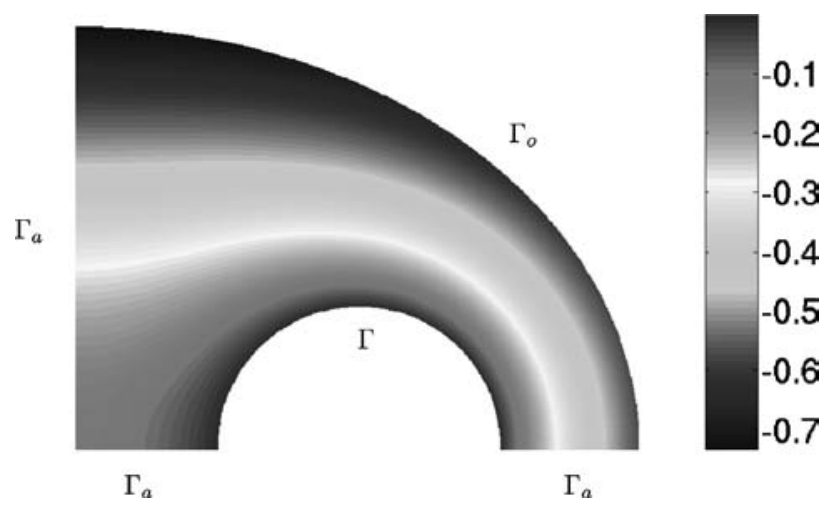

Figure 6. Part of the temperature distribution in a cylindrical insulation of two pipes for $r=1, u_{0}=0$, $u_{1}=-1$, and $\gamma=1$. 
Assuming that the insulation material is homogeneous, the temperature distribution $u$ satisfies

$$
\begin{aligned}
& \Delta u=0 \quad \text { in } D=D_{\mathrm{c}} \cap\left\{x: x_{v}>0\right\}, \\
& u=u_{0} \quad \text { on } \Gamma \text {, } \\
& \partial_{\perp} u=-\gamma\left(u-u_{1}\right) \quad \text { on } \Gamma_{o} \text {, } \\
& \partial_{\perp} u=0 \quad \text { on } \Gamma_{a} \text {, }
\end{aligned}
$$

where $\partial_{\perp}$ denotes the normal derivative, $u_{0}$ and $u_{1}$ are the temperatures of the pipes and the surrounding medium, respectively, and $\gamma$ is a material constant. The last equation is an artificial boundary condition, reflecting the symmetry of the solution, which needs to be computed only in a quarter of the cross section $D_{\text {c }}$.

The concrete values of the parameters are not particularly relevant since we can translate and scale the solution $u$ as well as the variables $x$. A convenient choice is given in the figure. For these data

$$
H_{\Gamma}^{1}=\left\{u \in H^{1}: u_{\mid \Gamma}=0\right\}
$$

is the appropriate Hilbert space $H$ for the variational formulation, noting that the essential boundary conditions are homogeneous.

The weak form of the differential equation is obtained in the usual way. Multiplying by $v \in H_{\Gamma}^{1}$ and integrating by parts,

$$
0=-\int_{D} \Delta u v=\int_{D} \operatorname{grad} u \operatorname{grad} v-\int_{\Gamma_{o} \cup \Gamma_{a}} \partial_{\perp} u v .
$$

Eliminating $\partial_{\perp} u$ with the aid of the natural boundary conditions, we see that

$$
a(u, v)=\int_{D} \operatorname{grad} u \operatorname{grad} v+\int_{\Gamma_{o}} u v, \quad \lambda(v)=-\int_{\Gamma_{o}} v
$$

are the bilinear and the linear form for the energy functional (1). The ellipticity of $a$ follows from the Poincaré-Friedrichs inequality which yields the lower bound of the norm equivalence

$$
\|u\|_{1}^{2} \asymp a(u, u), \quad u \in H_{\Gamma}^{1} .
$$

The continuity of $\lambda$ is a consequence of the trace theorem, asserting the boundedness of the restriction $\left.u \mapsto u\right|_{\Gamma}: H^{1}(D) \rightarrow L_{2}(\Gamma)$. Hence, the error estimate (8) is valid with $\ell=1$.

We computed finite element approximations $u_{h}$ with the standard hat-function basis and Web-splines of degree $n=1, \ldots, 5$. Figure 7 compares the two types of discretizations. Despite its simplicity, the domain does not permit a regular triangulation. In contrast, the Web-method takes advantage of the elementary boundary curves. We can choose

$$
w(x)=\left(x_{1}-2 r\right)^{2}+x_{2}^{2}-r^{2}
$$

as weight function for the finite element subspace $w^{e} \mathbb{B}_{h} \subset H_{\Gamma}^{1}$. The figure also illustrates that only in a neighborhood of the curved boundaries inner B-splines $b_{i}$ have to 

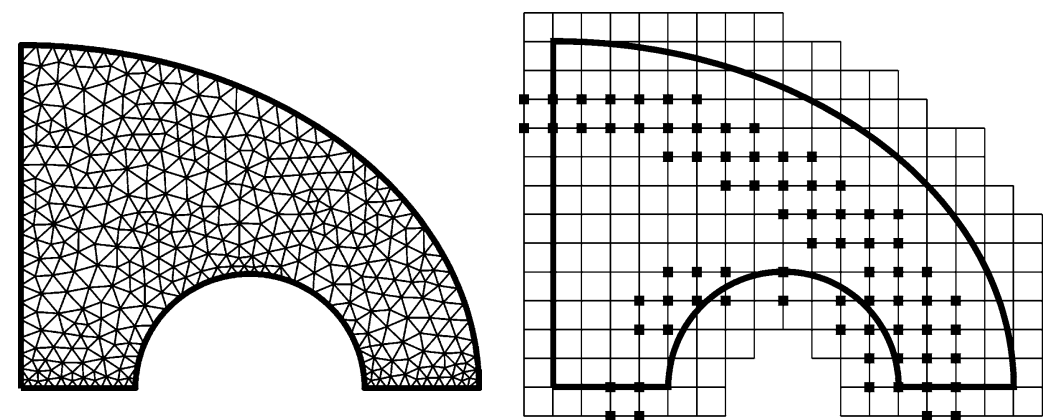

Figure 7. Triangulation for standard mesh-based elements (left) and discretization with bilinear Web-splines (right).
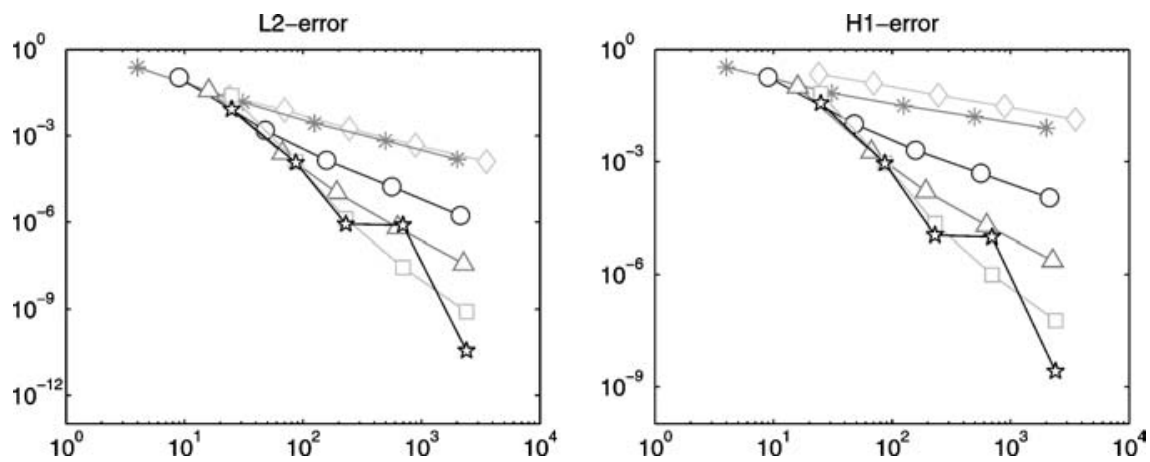

Figure 8. Relative errors for hat-functions $(\diamond)$ and Web-spline approximations of degree $n=1,2,3,4,5$ (markers $*, \circ, \triangle, \square, \star$ ) as a function of the dimension $d$.

be extended (marked with squares). For B-splines in the interior of $D$ or adjacent to the straight boundaries, the coefficients $e_{i, k}$ in definition 1 with $k \neq i$ are zero. Hence, as $h$ becomes small, the Web-space is almost a standard weighted spline space.

Figure 8 confirms the typical error behavior

$$
\left\|u-u_{h}\right\|_{0} \preceq h^{n+1}, \quad\left\|u-u_{h}\right\|_{1} \preceq h^{n},
$$

noting that $d \asymp h^{-2}$. Since the solution is smooth, the second estimate follows from (8), recalling that $\|\cdot\|_{1}$ is a norm on $H_{\Gamma}^{1}$. The additional factor $h$ for the $L_{2}$-norm is obtained via the Aubin-Nitsche duality principle (cf., e.g., [4]). The numerical results show that using higher degree pays off. For the discretizations shown in figure 7, the $L_{2}$-error of Web-approximations of degree 3 is by a factor 100 smaller than the error for standard finite elements, although less basis functions are used. Moreover, the accuracy of standard mesh-based approximations is limited by the required size of the linear systems.

Figure 9 shows further error statistics. Unlike for standard continuous finite elements, we can compute the pointwise residual of the differential equation for Web- 

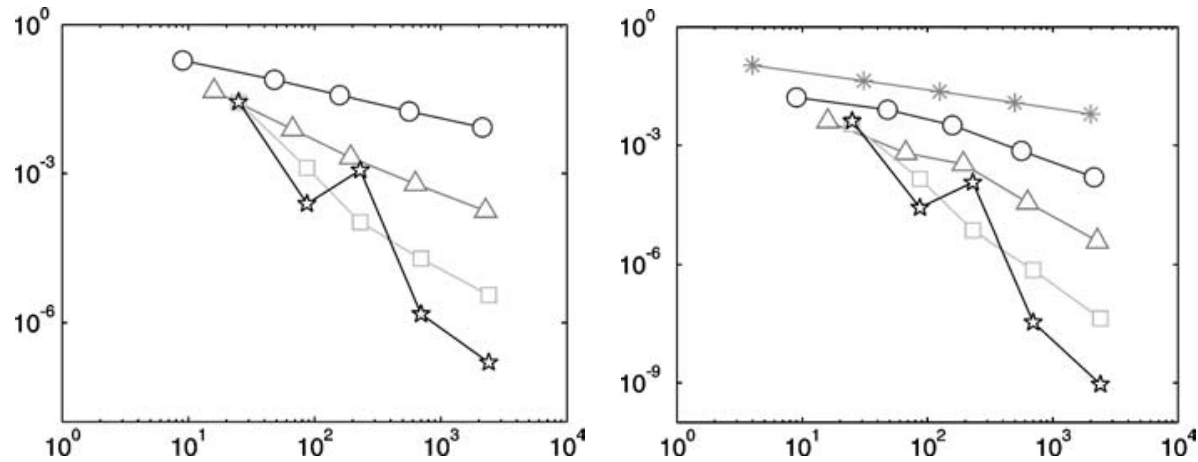

Figure 9. Residual $e_{r}=\max _{x \in D}\left|\Delta u_{h}(x)\right|$ (left) and boundary error $e_{b}=\max _{x \in \Gamma_{a}}\left|\partial_{\perp} u_{h}(x)\right|$ (right).

approximations of degree $n>1$. Even though we used the stronger maximum norm, the numerical results indicate optimal rates:

$$
e_{r} \preceq h^{n-1} .
$$

This is also the case for the artificial boundary condition. We have

$$
e_{b} \preceq h^{n}
$$

in accordance with the error of spline quasi-interpolants. Both of the above max-norm estimates are not covered by standard finite element theory. More research on the Webmethod is required to fully understand the precise error behavior in norms which are not directly related to the variational approach.

\section{Linear elasticity}

Numerical continuum mechanics has been the starting point for the development of finite element methods. In this application, finite elements can be interpreted as small building blocks of the elastic material. The basic physical model is illustrated in figure 10. An elastic solid, fixed at a portion $\Gamma$ of its boundary, is deformed under volume and surface forces with densities $\left(f_{1}, f_{2}, f_{3}\right)$ and $\left(g_{1}, g_{2}, g_{3}\right)$, respectively. The resulting displacement

$$
u\left(x_{1}, x_{2}, x_{3}\right)=\left(u^{1}(x), u^{2}(x), u^{3}(x)\right), \quad x \in D,
$$

is typically very small. Large distortions indicate excessive forces which cannot be adequately computed with a linear model.

The variational formulation follows directly from physical principles [17]. The stationary displacement $u$ minimizes the total energy

$$
Q(u)=\frac{1}{2} \underbrace{\int_{D} \sigma(u) \varepsilon(u)}_{a(u, u)}-(\underbrace{\int_{D} f u+\int_{\partial D \backslash \Gamma} g u}_{\lambda(u)})
$$




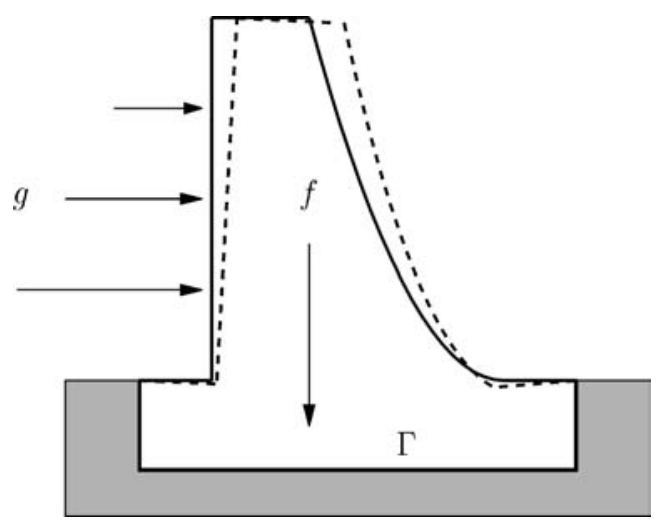

Figure 10. Deformation of an elastic solid under internal and boundary forces.

( $\left.\sigma \varepsilon=\sum_{k, \ell} \sigma_{k, \ell} \varepsilon_{k, \ell}\right)$. In the first integral, $\varepsilon$ denotes the strain and $\sigma$ the stress tensor, defined by

$$
\varepsilon_{k, \ell}(u)=\frac{1}{2}\left(\partial_{k} u^{\ell}+\partial_{\ell} u^{k}\right)
$$

and Hooke's law

$$
\sigma_{k, \ell}(u)=\lambda \operatorname{trace} \varepsilon(u) \delta_{k, \ell}+2 \mu \varepsilon_{k, \ell}(u) .
$$

The constants $\lambda$ and $\mu$ are the Lamé constants, which describe the elastic properties of the material.

As for the much simpler case of Laplace's equation, considered in the last section, the quadratic energy functional involves only first order partial derivatives. However, the admissible displacements are vector valued functions

$$
u=\left(u^{1}, u^{2}, u^{3}\right) \in\left(H_{\Gamma}^{1}\right)^{3} .
$$

Accordingly, the Web-approximation is of the form

$$
u_{h}=\sum_{i}\left(u_{i}^{1}, u_{i}^{2}, u_{i}^{3}\right) B_{i} \in\left(w^{e} \mathbb{B}_{h}\right)^{3},
$$

with a weight function $w$ which vanishes on $\Gamma$.

It is easily seen that $a$ and $\lambda$ are continuous:

$$
|a(u, v)| \preceq\|\| u\|\|_{1}\|v\|_{1}, \quad|\lambda(u)| \preceq\|u\|_{1},
$$

where \|\|$\cdot\|\|_{1}$ denotes the product norm on $\left(H_{\Gamma}^{1}\right)^{3}$. The lower bound for $a$, required for ellipticity, is much harder to prove. It involves Korn's inequality

$$
\|u\|_{0}^{2}+\int_{D} \varepsilon(u) \varepsilon(u) \succeq\|u\|_{1}^{2}
$$

(cf. [18] for a rigorous derivation). 

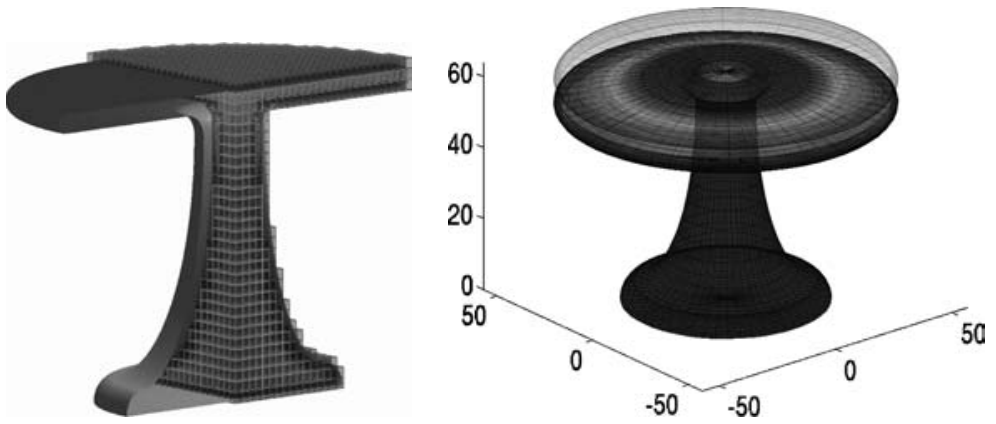

Figure 11. Grid of Web-spline approximation (left) and principal stress (right) of a table deformed under its weight.

Table 1

Statistics of the cell types and Web-basis for different grid widths $h$ $\left(r_{t}\right.$ denotes the radius of the tabletop).

\begin{tabular}{rrrrrr}
\hline$h / r_{t}$ & inner cells & bnd cells & inner spl & outer spl & unextended \\
\hline 0.08 & 338 & 1930 & 998 & 3986 & 6 \\
0.04 & 5596 & 7634 & 13070 & 9258 & 1037 \\
0.02 & 52497 & 30290 & 82435 & 33538 & 20493 \\
\hline
\end{tabular}

Figure 11 shows an example. A stone table $\left(\lambda=1.1 \times 10^{6} \mathrm{~N} / \mathrm{cm}^{2}, \mu=\right.$ $\left.2.7 \times 10^{6} \mathrm{~N} / \mathrm{cm}^{2}\right)$ is fixed at the bottom $\Gamma$ and subjected to its own weight $(f=$ $2.4 \times 10^{-2} \mathrm{~N} / \mathrm{cm}^{3}$ ). As is to be expected, the principal stress

$$
\sigma_{p}=\|\sigma\|
$$

is largest in the middle of the tabletop.

In this example,

$$
\Gamma=\left\{x: x_{1}^{2}+x_{2}^{2} \leqslant r^{2}, x_{3}=0\right\}, \quad w(x)=x_{3} .
$$

Such a trivial choice of the weight function is typical for many applications in elasticity. While the objects may have a complicated shape, the fixed portion $\Gamma$ of the boundary is often planar or permits at least a simple analytic description.

The numerical solution was computed with cubic Web-splines, and a few statistics of the approximation are listed in table 1 . Since the domain $D$ is relatively thin, the percentage of boundary cells is initially quite large. Hence, the majority of inner B-splines have to be extended. However, as will be shown in section 7, the linear combinations $\sum_{k} e_{i, k} b_{k}$ can be efficiently generated during the assembly of the Ritz-Galerkin matrix.

\section{Thin plates}

For many important applications, the linear elasticity model admits a dimension reduction. An example is Kirchhoff's theory of thin plates of uniform thickness $d$ which 
is small compared to the horizontal dimensions (cf., e.g., [17]). Here, it is assumed that for a transversal force the vertical displacement is independent of $x_{3}$ :

$$
u^{3}(x)=u\left(x_{1}, x_{2}\right), \quad\left(x_{1}, x_{2}, x_{3}\right) \in D \times\left[-\frac{d}{2}, \frac{d}{2}\right] .
$$

Moreover, the third components of the stress tensor are neglected: $\sigma_{3,1}=\sigma_{3,2}=\sigma_{3,3}$ $=0$. By Hooke's law (11), this implies

$$
u^{1}(x)=-x_{3} \partial_{1} u, \quad u^{2}(x)=-x_{3} \partial_{2} u, \quad \varepsilon_{3,3}=-\frac{\lambda}{\lambda+2 \mu}\left(\varepsilon_{1,1}+\varepsilon_{2,2}\right) .
$$

Writing out the remaining horizontal components of the stress/strain relation in detail,

$$
\left(\begin{array}{l}
\sigma_{1,1} \\
\sigma_{2,2} \\
\sigma_{1,2}
\end{array}\right)=\frac{E}{1-v^{2}}\left(\begin{array}{ccc}
1 & v & 0 \\
v & 1 & 0 \\
0 & 0 & 1-v
\end{array}\right)\left(\begin{array}{l}
\varepsilon_{1,1} \\
\varepsilon_{2,2} \\
\varepsilon_{1,2}
\end{array}\right),
$$

where $E=\mu(3 \lambda+2 \mu) /(\lambda+\mu)$ is the Young modulus and $v=\lambda /(2(\lambda+\mu))$ the Poisson ratio. With the aid of these identities, all quantities in the first integral of the energy functional (9) can be expressed in terms of second derivatives of the vertical displacement $u$. Integrating over $x_{3} \in[-d / 2, d / 2]$, a lengthy but straightforward computation yields

$$
Q(u)=\frac{\gamma}{2} \int_{D}(\Delta u)^{2}+2(1-v)\left[\left(\partial_{1} \partial_{2} u\right)^{2}-\left(\partial_{1}^{2} u \partial_{2}^{2} u\right)\right]-\int_{D} f u
$$

with $\gamma=E d^{3} /\left(12\left(1-v^{2}\right)\right)$ and $f$ the density of a force normal to the plate (combining the contributions from external and internal forces in (9)).

The variational formulation becomes particularly simple for a clamped plate, corresponding to the boundary conditions

$$
\left.u\right|_{\partial D}=\left.\partial_{\perp} u\right|_{\partial D}=0 .
$$

In this case, integration by parts shows that the term in brackets disappears, and the bilinear form equals

$$
a(u, v)=\gamma \int_{D} \Delta u \Delta v
$$

which is elliptic on $H_{0}^{2}(D)$.

For a conforming Ritz-Galerkin approximation, Web-splines of degree $\geqslant 2$ are needed. The weight function must vanish to second order at the boundary,

$$
w(x) \asymp \operatorname{dist}(x, \partial D)^{2},
$$

and is constructed simply by squaring the standard weight functions, described in section 2.

Figure 12 visualizes the numerical solution for a plate, clamped along a free-form boundary and subjected to gravitation. The left graphic shows the resulting deformation, 

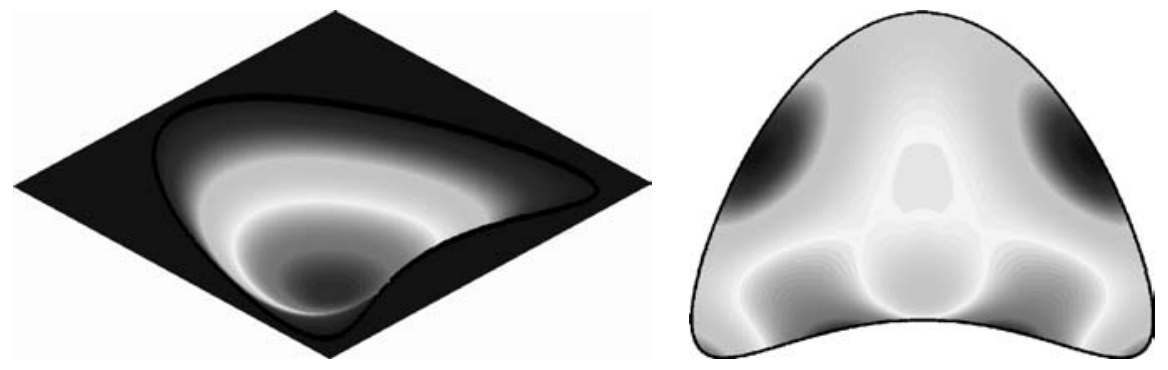

Figure 12. Displacement (left) and von Mises stress (right) for a clamped plate.
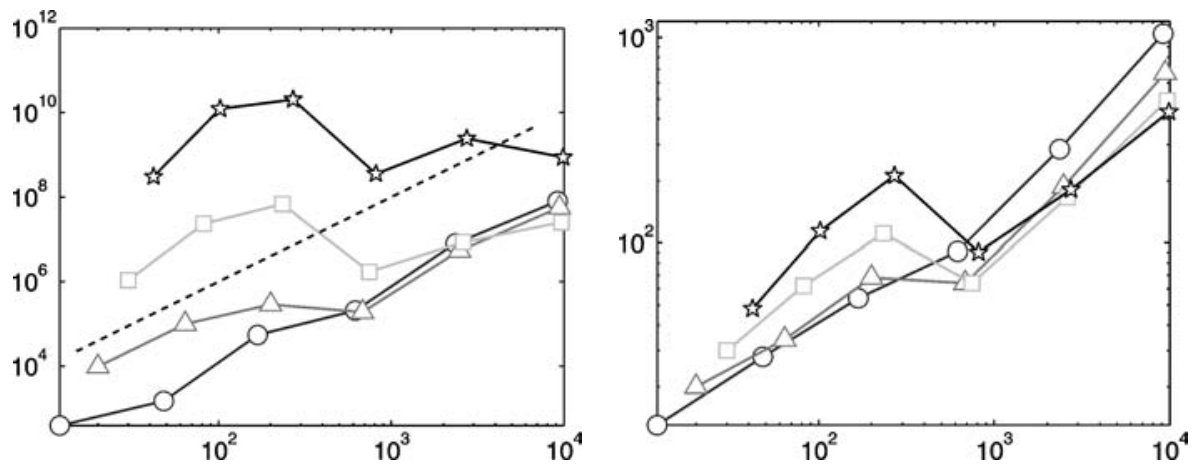

Figure 13. Condition of the Ritz-Galerkin system (left) and number of pcg-iterations (right) versus the dimension $d$ of the Web-space for degree $n=2, \ldots, 5$ (markers $\circ, \triangle, \square, \star$ ).

and on the right side we see the distribution of the von Mises stress $\sigma_{m}$, which is defined as

$$
\sigma_{m}=\sqrt{\bar{\sigma}_{1}^{2}+\bar{\sigma}_{2}^{2}-\bar{\sigma}_{1} \bar{\sigma}_{2}}
$$

where $\bar{\sigma}_{1}, \bar{\sigma}_{2}$ are the eigenvalues of the two-dimensional stress tensor $\sigma$. We notice that $\sigma_{m}$ is largest near the concave portion of the boundary at the bottom.

For fourth order problems the stabilization of the B-splines via the extension procedure is of even greater importance. As is well known, standard conforming finite element subspaces for the plate problem have

$$
\text { cond } G_{h} \asymp h^{-4} \text {. }
$$

Hence, keeping outer B-splines with very small support in $D$ in the basis leads to excessively ill-conditioned Ritz-Galerkin systems. For the stabilized Web-splines, the standard estimate (12) remains valid and iterative solvers perform reasonably well.

Figure 13 shows a few statistics for the example of figure 12. The condition numbers on the left side are in good agreement with the predicted rate $\mathrm{O}\left(h^{-4}\right) \asymp d^{2}$, shown as a dashed line. It is perhaps somewhat surprising that $\operatorname{cond} G_{h}$ does not grow substantially with the degree $n$. For the smallest grid width, the values differ by less than a factor 50. The robustness of the Web-method with respect to the chosen degree is also 
apparent from the number of pcg-iterations. Moreover, given the large condition of the Ritz-Galerkin matrix, the computing times, shown on the right side of the figure, remain acceptable. To solve the system with a relative accuracy of $10^{-8}$, roughly $d / 10$ iterations are required.

The plate problem can be viewed as a simple test case for modeling elastic shells [5]. Here, Koiter's classical model, which requires continuously differentiable elements, can be effectively implemented using Web-splines. Unlike for conforming mesh-based elements, increasing smoothness does not present any problems. It is conceivable that the Web-method provides a good alternative to modern non-conforming techniques, similarly as the new subdivision methods proposed in [6].

\section{Implementation}

In this section we comment briefly on several implementation aspects. Conceptually, the Web-method is similar to standard finite element techniques - we just use a different type of basis functions. However, as is to be expected, the tensor product grid offers a number of computational advantages. We discuss each of the basic algorithmic components in turn (cf. [10] for details).

Matrix assembly. We first note that the extension procedure can be conveniently combined with the assembly of the Ritz-Galerkin matrix. By linearity,

$$
a\left(B_{i^{\prime}}, B_{i}\right)=\sum_{k^{\prime}} \sum_{k} e_{i^{\prime}, k^{\prime}} a\left(w b_{k^{\prime}}, w b_{k}\right) e_{i, k} .
$$

Hence, the stabilization amounts to two sparse matrix multiplications (the matrix $E$ has few off-diagonal entries).

As is common practice, the entries

$$
a\left(w b_{k^{\prime}}, w b_{k}\right)=\sum_{Q} \int_{Q \cap D} \varphi\left(w, b_{k^{\prime}}, b_{k}, \ldots\right)
$$

are computed by adding the contributions from each grid cell $Q$. This involves Gauß quadrature of functions $\varphi$ which depend on values and derivatives of the weight function, B-splines, and coefficients of the differential equation. Of course, B-splines can be handled very efficiently. This is also the case for the different types of weight functions. Rvachev's R-functions can be evaluated with the aid of automatic differentiation, which is ideally suited for the recursive algorithmic definition of $w$. Numerical weight functions are constructed in terms of distance functions. While Newton's method must be used, this does require only few iterations. Since we are processing neighboring quadrature points, very good starting guesses are available. Furthermore, we note that the normal derivative of $\operatorname{dist}(\cdot, \partial D)$ equals 1 which facilitates the computation of gradients.

To avoid a loss of accuracy (as would be the case when using polygonal approximations), integrals over boundary grid cells require a special treatment. The integration 
techniques are similar to methods for isoparametric elements. Based on the intersection pattern of $\partial D$ with $\partial Q$, we subdivide each set $Q \cap D$ into subcells which can be mapped smoothly to standard domains (squares or cubes, triangles or tetrahedra, etc.). The numerical transformations serve to determine the appropriate location of the Gauß points. They only need to be as accurate as required by the selected approximation order. Figure 14 gives two examples. While two-dimensional subdivision is straightforward, cell subdivision in three dimensions requires more sophisticated algorithms. Fortunately, the portion of more complicated cases, like corners and edges within the grid cell, is asymptotically small (by two orders of magnitude with respect to $1 / h$ ). Hence, the overall computational complexity is not significantly affected by the necessary subdivision procedures.

Multigrid solvers. Given the high accuracy of the Web-method, the Ritz-Galerkin systems are relatively small and thus can be solved effectively with preconditioned conjugate gradient iterations. Even for ill-conditioned fourth order problems, solving the linear systems is not too time-consuming (cf. figure 13 of the last section). Hence, for Web-splines multigrid solvers are primarily important for large scale three-dimensional applications and time-dependent problems.

Of course, a regular grid is ideally suited for multilevel schemes since subdivision provides a natural grid transfer operator. We can express a B-spline $b_{\ell}^{\prime}$ on a coarse grid with width $2 h$ as a linear combination

$$
b_{\ell}^{\prime}=\sum_{k} s_{2 k-\ell} b_{k} \quad s_{\alpha}=2^{-n m} \prod_{\nu=1}^{m}\left(\begin{array}{c}
n+1 \\
\alpha_{\nu}
\end{array}\right)
$$

(cf., e.g., [7]). Hence, for weighted splines on consecutive grid levels,

$$
\sum_{k^{\prime} \in K^{\prime}} u_{k^{\prime}}^{\prime}\left(w b_{k^{\prime}}^{\prime}\right)=\sum_{k \in K} u_{k}\left(w b_{k}\right), \quad U=S U^{\prime}
$$
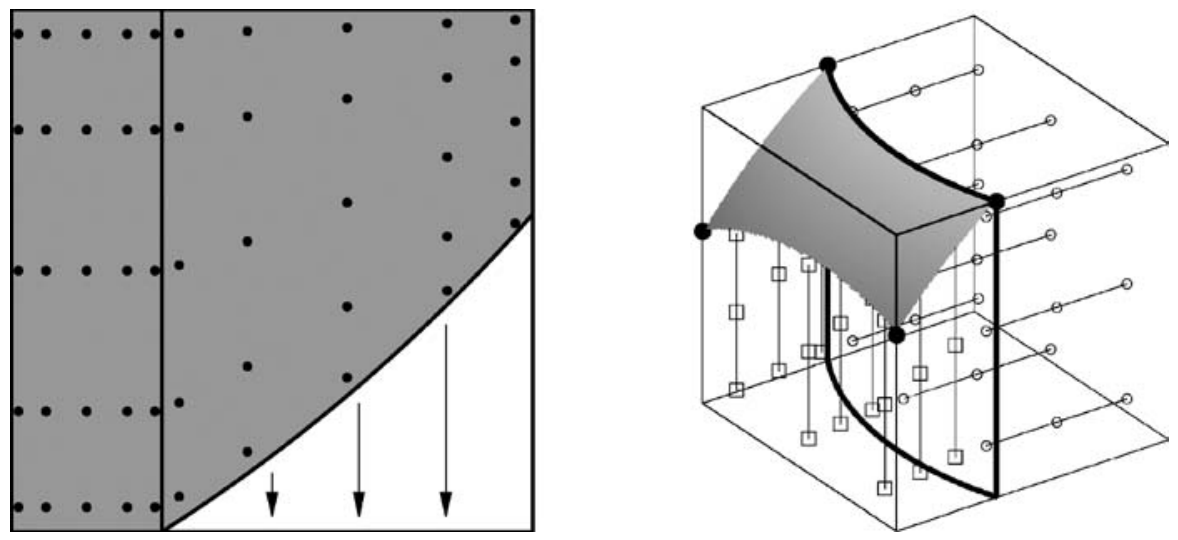

Figure 14. Partition of boundary grid cells and Gauß points for numerical integration. 


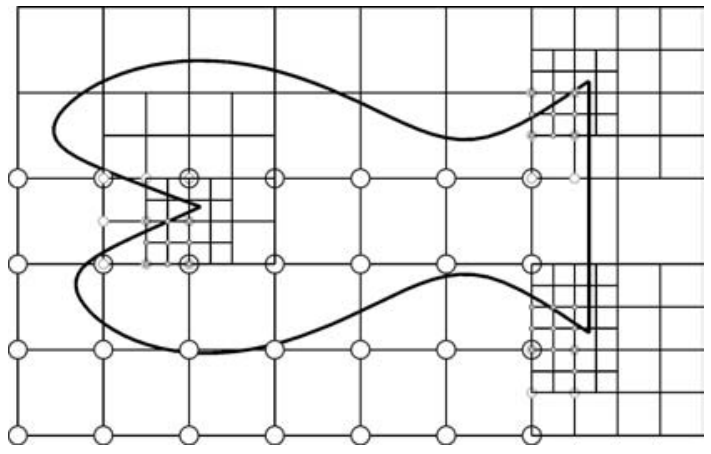

Figure 15. Adaptive refinement for bilinear weighted B-splines $w b_{k}$ (marked with circles at the lower left corner of their support) near corners of a two-dimensional domain.

with a sparse matrix $S$. As is well known, the grid transfer matrix $S$ is all that is needed for implementing a standard finite element multigrid scheme.

Programming the iteration step for the Web-spaces $w^{e} \mathbb{B}_{h}$ is slightly more difficult since these spaces are not nested. We resolve this problem with the aid of the canonical projector (5), i.e., we use $P_{h} B_{i}^{\prime}$ as fine grid approximation of coarse grid Web-splines $B_{i}^{\prime}$. This provides again a sparse grid transfer matrix with simple explicitly defined entries.

Because of the stability of the Web-basis, the standard multigrid theory can be applied. For example, it can be shown without too much effort that the w-cycle converges with a grid independent rate, even though the Web-spaces are not nested [14].

Adaptive refinement. Web-splines can be defined more generally for arbitrary knot sequences [11]. However, unlike in one dimension, knot refinement has a global effect. For truly local subdivision, hierarchical spline spaces should be used.

The natural concept of nested B-spline bases is illustrated in figure 15 for the weighted spline spaces $w \mathbb{B}_{h}$. In a critical portion of the region $D$, where large errors are to be expected, we replace the B-splines $b_{k}$ by the B-splines with grid width $h / 2$. This procedure is applied recursively, i.e., the resulting space is spanned by weighted B-splines on a sequence of grids with widths $h, h / 2, h / 4, \ldots$. Some care is required in order to maintain linear independence, which does not, however, lead to any significant geometric restrictions [10].

There are a number of variants of hierarchical spline spaces, in particular in computer graphics and scattered data fitting. Most recently, the technique has been very successfully applied in the finite element simulation of elastic shells [6]. Compared to wavelets, the implementation is relatively straightforward, which often outweighs the slight lack of stability with respect to many grid levels.

\section{Concluding remarks}

The applications discussed in this paper show that Web-splines can provide a good alternative to standard finite element approximations. In particular, the following fea- 
tures of the Web-method should be noted:

- No mesh generation is required.

- Boundary conditions are represented exactly.

- Accurate numerical solutions are possible with relatively low-dimensional subspaces.

- Smoothness and approximation order can be chosen arbitrarily.

- Regardless of the degree, each grid point corresponds to one basis function.

- Hierarchical bases permit adaptive refinement und multigrid algorithms.

In effect, Web-splines combine the advantages of B-splines and standard meshbased elements. Hence, they bridge the gap between geometric modeling and numerical simulation, two fields which are very closely linked in many engineering applications.

\section{Acknowledgements}

We thank J. Hörner for contributing the example in figure 11 and his numerical integration package as well as J. Wipper, one of the co-founders of the Web-method, for his involvement in our Web-software projects.

\section{References}

[1] J.H. Argyris, Energy Theorems and Structural Analysis (Butterworths, London, 1960).

[2] T. Belytschko, Y. Krongauz, D. Organ, M. Fleming and P. Krysl, Meshless methods: An overview and recent developments, Comput. Methods Appl. Mech. Engrg. 139(1-4) (1996) 3-47.

[3] P. Bochev and M. Gunzburger, Finite element methods of least squares type, SIAM Rev. 40 (1998) 789-837.

[4] P.G. Ciarlet, The Finite Element Method for Elliptic Problems (North-Holland, Amsterdam, 1978).

[5] P.G. Ciarlet, Mathematical Elasticity, Vol. 3: Theory of Shells (North-Holland, Amsterdam, 2000).

[6] F. Cirak, M. Ortiz and P. Schröder, Subdivision surfaces: A new paradigm for thin-shell finite-element analysis, Internat. J. Numer. Methods Engrg. 47 (2000) 2039-2072.

[7] E. Cohen, R.F. Riesenfeld and G. Elber, Geometric Modeling with Splines: An Introduction (A.K. Peters, 2001)

[8] C. de Boor, A Practical Guide to Splines (Springer, Berlin, 1978).

[9] K. Höllig, Finite element approximation with splines, in: Handbook of Computer Aided Geometric Design, eds. G. Farin, J. Hoschek and M.S. Kim (Elsevier, Amsterdam, 2002) pp. 283-308.

[10] K. Höllig, Finite Element Methods with B-Splines, Frontiers in Applied Mathematics, Vol. 26 (SIAM, Philadelphia, PA, 2003).

[11] K. Höllig and U. Reif, Nonuniform Web-splines, Computer Aided Geometric Design 20 (2003) 277294.

[12] K. Höllig, U. Reif and J. Wipper, Weighted extended B-spline approximation of Dirichlet problems, SIAM J. Numer. Anal. 39(2) (2001) 442-462.

[13] K. Höllig, U. Reif and J. Wipper, Verfahren zur Erhöhung der Leistungsfähigkeit einer Computereinrichtung bei Finite-Elemente-Simulationen und eine solche Computereinrichtung, Deutsche Patentschrift DE 10023377 C2 (2003).

[14] K. Höllig, U. Reif and J. Wipper, Multigrid methods with Web-splines, Numer. Math. 91(2) (2002) 237-256. 
[15] K. Höllig, U. Reif and J. Wipper, Process for increasing the efficiency of a computer in finite element simulations and a computer for performing that process, United States Patent Application, US2002/0029135A1 (2002).

[16] L.W. Kantorowitsch and W.I. Krylow, Näherungsmethoden der Höheren Analysis (VEB Deutscher Verlag der Wissenschaften, Berlin, 1956).

[17] L.D. Landau and E.M. Lifshitz, Theory of Elasticity, 3rd ed. (Pergamon Press, Elmsford, NY, 1986).

[18] J.A. Nitsche, On Korn's second inequality, RAIRO J. Numer. Anal. 15 (1981) 237-248.

[19] V.L. Rvachev and T.I. Sheiko, R-functions in boundary value problems in mechanics, Appl. Mech. Rev. 48(4) (1995) 151-188.

[20] L.L. Schumaker, Spline Functions: Basic Theory (Wiley-Interscience, New York, 1980).

[21] Special Issue on Meshless Methods, Comput. Mech. Appl. Mech. Engrg. 139 (1996).

[22] E.M. Stein, Singular Integrals and Differentiability Properties of Functions (Princeton Univ. Press, Princeton, 1970).

[23] G. Strang and G.J. Fix, An Analysis of the Finite Element Method (Prentice-Hall, Englewood Cliffs, NJ, 1973).

[24] M.J. Turner, R.W. Clough, H.C. Martin and L.C. Topp, Stiffness and deflection analysis of complex structures, J. Aeronaut. Sci. 23(9) (1956) 805-823, 854.

[25] O.C. Zienkiewicz and R.I. Taylor, Finite Element Method, Vols. I-III (Butterworth \& Heinemann, 2000). 